\title{
Norois
}

Environnement, aménagement, société

197 | 2005/4

Varia

\section{L'agro-alimentaire chez les géographes des campagnes en France au xxe siècle}

Agri-food and french rural geographers (20th century)

\section{Christine Margetic}

\section{CpenEdition}

\section{Journals}

Édition électronique

URL : http://journals.openedition.org/norois/301

DOI : $10.4000 /$ norois.301

ISBN : 978-2-7535-1545-1

ISSN : $1760-8546$

Éditeur

Presses universitaires de Rennes

Édition imprimée

Date de publication : 1 décembre 2005

Pagination : $37-53$

ISBN : 978-2-7535-0238-3

ISSN : 0029-182X

\section{Référence électronique}

Christine Margetic, «L'agro-alimentaire chez les géographes des campagnes en France au xxe

siècle », Norois [En ligne], 197 | 2005/4, mis en ligne le 15 décembre 2008, consulté le 30 avril 2019

URL : http://journals.openedition.org/norois/301 ; DOI : 10.4000/norois.301

Ce document a été généré automatiquement le 30 avril 2019.

(c) Tous droits réservés 


\title{
L'agro-alimentaire chez les géographes des campagnes en France au xxe siècle
}

\author{
Agri-food and french rural geographers (20th century)
}

\author{
Christine Margetic
}

\section{NOTE DE L'ÉDITEUR}

Cet article a été reçu le 9 juin 2005 et définitivement accepté le 2 décembre 2005.

1 «Si chacun s'accorde à souligner l'ampleur des mutations techniques de l'agriculture depuis quelques décennies, les considérations sur les liaisons entre le secteur primaire et les autres secteurs économiques ont moins retenu l'attention » soulignait J.-P. Diry en 1997. Parmi les autres secteurs économiques figure pourtant le secteur agro-alimentaire, réceptacle traditionnel et «naturel » de la grande majorité des volumes de matières premières agricoles commercialisées. Est-il réellement absent des travaux des géographes, notamment chez les géographes des campagnes?

2 Cet article $^{1}$ vise à répondre - partiellement - à cette question selon une double perspective. En premier lieu, la relecture des auteurs va privilégier une approche diachronique des préoccupations sur la question de la transformation alimentaire des matières premières agricoles. Objet d'étude géographique, objet géographique ou composante d'un système spatialisé, les industries agro-alimentaires (IAA) forment un ensemble hétérogène, ce qui influe sur leur degré d'appropriation par les auteurs qui abordent l'analyse des liens entre les différents mécanismes de structuration territoriale fondée sur les produits agricoles. En second lieu, cette relecture va tenter de comprendre leurs formes de territorialité, et de cerner leur rôle dans la qualification des territoires à partir de l'analyse de leurs pratiques spatiales. 
Ce premier bilan repose sur l'exploitation d'articles et de thèses de géographes ruralistes à partir du début du xxe siècle, ce qui correspond à la phase d'individualisation des firmes vis-à-vis de la ferme, sur la base d'un double parti pris méthodologique. D'une part, seules sont abordées les IAA au contact du monde agricole, hors vin ${ }^{2}$. D'autre part, ce travail s'appuie sur des tableaux antérieurs de la production française ${ }^{3}$ et sur une sélection d'articles et de thèses considérés comme représentatifs quant aux angles de la recherche rurale.

4 L'article est organisé autour des trois phases d'appropriation des firmes agroindustrielles par les géographes ruralistes français : d'une relative indifférence pour des activités individualisées au travers des matières premières transformées, on passe à partir des années 1960 à une phase de caractérisation d'un secteur essentiel pour le milieu rural, ces industries étant parallèlement considérées en tant que sous-secteur fonctionnel d'un système spatialisé en voie de mutation depuis les années 1990.

\section{L'agro-alimentaire au travers du prisme des activités agricoles}

5 De la fin du xixe siècle jusqu'à l'entre-deux-guerres, on assiste à une transition marquée de structures proto-industrielles encore attachées à la ferme à l'affirmation d'industries qui travaillent des matières premières agricoles, la Première Guerre mondiale servant de catalyseur, puis les années 1960 d'accélérateur. Activités en devenir, se démarquent alors certaines d'entre elles dans le contexte d'agrocentrisme des travaux relevant de l'école française de géographie (1870-1914), puis de géographie humaine de la période classique de la discipline (1918-1968) (Berger et al., 1975). De manière générale, elles sont envisagées en tant qu'identificateur d'une économie rurale ou agricole.

\section{Des « industries agricoles » pour Maximilien Sorre}

6 «Il est bien difficile d'ignorer les relations strictes qui unissent l'agriculture aux autres formes de l'activité économique. Mais on en méconnaît parfois l'importance », alors que, dans les années 1920, « l'agriculture fournit à l'industrie des masses énormes de produits bruts » (Demangeon, 1929). Le singulier employé est explicite du regard que leur portent alors les géographes, qui ciblent avant tout des catégories d'industries (sucrerie, laiterie, etc.) sans les associer nécessairement.

Le premier à employer l'expression « industries agricoles » est M. Sorre (1929). Titulaire de la chaire de géographie à Lille entre 1921 et 1931, il privilégie une approche réflexive qui reflète ses liens avec les acteurs de la vie économique régionale, notamment la Première région économique, institution regroupant les chambres de Commerce et d'Industrie du Nord-Pas-de-Calais. Ainsi, il associe « avec insistance les activités agricoles à la vie urbaine et industrielle de la région [...] L'esprit en est systémique : si le livre commence par l'examen des conditions naturelles, il les relie aussitôt aux transformations des pays et des sociétés, à la dynamique économique, aux marchés... Le bilan insiste sur les liens entre l'agriculture, l'industrie et la ville et débouche sur une prospective » (Thumerelle, 1998). Une belle géographie appliquée loin des stéréotypes de la géographie régionale. 
8 Pour M. Sorre (1929), ces « industries, dont l'objet est la transformation des produits agricoles régionaux, dans la plupart des cas en vue de l'alimentation, parfois aussi pour la satisfaction d'autres besoins ", présentent toujours un lien au lieu régional, ce qui écarte les huileries par exemple. Ainsi, il aborde "la meunerie et les industries qui s'y rattachent» (4 pages), "sucrerie, distillerie et industries annexes» (16 pages), «brasserie, malterie, cidrerie » (5 pages), et « autres industries agricoles, la chicorée, les produits du lait, les conserves » (5 pages). Que la moitié du chapitre renvoie aux seules sucreries et au raffinage peut surprendre, mais l'historien P. Veylet (1997) y voit le fait que les structures productives de ce secteur associé à la culture de betterave à sucre étaient déjà clairement identifiées, à la différence d'autres activités où il était souvent ardu de séparer les industries fermières ou rurales du monde agricole et de sa pluriactivité traditionnelle.

9 Le rôle d'acteur de l'économie rurale de ces firmes passe donc par le filtre d'une production agricole donnée, en général préexistante à la phase d'industrialisation de la transformation, sauf exception, comme pour la chicorée (Demangeon, 1905). Pourtant, si souvent «l'utilisation industrielle oriente la production agricole» (Sorre, 1929), ce point est rarement clairement explicité en tant que tel dans les travaux des géographes du début du xxe siècle.

\section{Des industries agricoles abordées en filigrane comme acteur « naturel » de la vie rurale}

10 La lecture de quelques thèses de Doctorat d'État et d'articles parus en particulier dans les Annales de Géographie des années 1920 à 1950, confirme l'existence d'un lien au lieu 4 . Par ordre d'importance sont abordées sucreries, activités liées au lait, industries des plantes grasses, et, plus accessoirement, conserveries, minoteries et industries liées aux céréales ou malteries, brasseries, etc.

11 Prenons les conserves de légumes. Elles sont pour M. Sorre (1929) l'exemple-type d'une industrie rigoureusement située dans les régions de production en raison de la nature très périssable des matières traitées, qui ne tolèrent pas un long transport. Leur développement se fait donc de manière privilégiée dans les zones maraîchères, ou à leur proximité, même si une partie des matières premières peut sortir du cadre de la proximité (épices de Marseille) (Flatrès, 1946).

12 Une même logique prévaut pour la sucrerie, exemple le plus abouti des préoccupations des géographes d'alors quant à l'identité d'une activité agro-industrielle. Dès l'introduction de sa thèse, A. Demangeon (1905) se réfère à leur présence pour délimiter la plaine de la craie vers l'est, et en portant sur une carte l'emplacement de toutes les fabriques, il parvient à circonscrire " presque exactement l'extension de la culture de la betterave", ce qui forme le cœur de sa réflexion. Pour expliquer son essor et sa concentration géographique dans le Nord de la France, il s'appuie sur des raisons naturelles - les qualités des sols - condition sine qua non mais non exclusive puisque comptent au moins pour autant des raisons économiques. En 1929, M. Sorre va plus loin : la cause initiale de la prospérité sucrière ne vient pas des modes de production, mais de facilités de communication (canaux et cours d'eau, chemin de fer), à l'origine d'un approvisionnement partiel en betteraves en Belgique et en Hollande jusqu'aux années 1930, à la proximité du bassin houiller, et, ce qui le démarque d'A. Demangeon, à l'initiative humaine symbolisée par de grands noms, Crespel, "père de la sucrerie 
indigène ", les familles Beghin et Say. Jouent aussi des éléments complètement exogènes (concurrence allemande, guerre, etc.) qui interagissent pour renforcer le positionnement nordiste.

En définitive, on peut dire qu'agriculture et industrie, et leurs évolutions propres, se nourrissent l'une l'autre. Et ce qui est frappant chez A. Demangeon, c'est finalement le caractère industriel qu'il attribue moins à la transformation de la betterave qu'à la culture même! Il n'aborde d'ailleurs pas l'industrie pour elle-même, mais plutôt comme une activité d'appoint destinée à soulager un surpeuplement rural aigu ou un sous-emploi saisonnier résultant $\mathrm{du}$ déséquilibre $\mathrm{du}$ calendrier agricole, et un débouché pour le monde agricole. En Picardie orientale, les interrelations sont alors étroites et parfaites entre ces deux acteurs, à la base d'un pays sucrier ébranlé par l'émergence de gros établissements en place de petites structures (Alexandre, 1928). Dès lors est posé un thème qui devient récurrent, celui de la réappropriation par le monde agricole de ce partenaire dont l'espace de référence transcende désormais les limites traditionnelles de la proximité.

\section{Des firmes agro-industrielles filles de la modernisation agricole}

La référence aux structures coopératives devient récurrente vers la fin des années 1920 , souvent par quelques lignes dans des écrits plutôt attachés à différencier des pays et leur vie rurale (Bobin, 1926 ; Dion, 1933 ; etc.), sauf ceux ayant trait aux fruitières (Delfosse, 1992 ; Ricard, 1993), ou ceux portant sur les effets de la révolution agricole qui tendent alors à se multiplier (Richard, 1929).

Plusieurs auteurs évoquent le contexte réactionnaire de leur création, lié à une baisse du prix du lait imposée par les ramasseurs (Dion, 1933) ou par de grosses laiteries industrielles issues d'un premier mouvement de concentration parfois éphémère (Renaudin, 1936). À l'inverse, d'autres appuient l'absence de sentiment coopératif comme dans le Bas-Boulonnais (Coudoux, 1957) ou plus souvent un caractère opportuniste pour certains adhérents, en lien avec une proximité géographique (Brunet, 1965) ou leurs logiques de modernisation (Dion, 1933) confortées par l'amélioration parallèle des axes routiers secondaires (Chevalier, 1952).

Géographie des sites de transformation et géographie agricole peuvent aller de pair. Les agriculteurs peuvent être tentés par une culture non connue précédemment mais réclamée par une activité agro-industrielle en émergence (pays du houblon autour d'Haguenau dès 1810) (Zeyl, 1930), l'opportunité pouvant disparaître en raison de facteurs conjoncturels au stade des industriels (froment Poulard en Limagne pour l'industrie clermontoise des pâtes alimentaires) (Arbos, 1932). À l'inverse, la disparition d'une culture locale n'induit pas toujours celle de l'outil industriel associé (survivance d'une importante huilerie à Arras malgré la disparition des oléagineux de la région) (Demangeon, 1905 ; Sorre, 1950a). Certaines agro-industries trouvent parfois de nouvelles zones d'expansion dans des espaces agricoles en reconversion, d'échelle variable (vignoble orléanais-blésois tourné vers la culture de légumes de plein champ à destination de l'industrie de la conserve alimentaire d'Orléans, voire du Mans) (Dion, 1933). Enfin, de manière plus indirecte, les agro-industries contribuent à la diversification d'espaces agricoles et à l'essor de productions annexes liées à la mise à disposition de sous-produits (petit-lait et progrès de l'élevage du porc en Gâtine) (Bobin, 1926). Nourrissant d'étroits rapports entre l'usine et le cultivateur, ces pratiques contribuent à 
l'amélioration constatée des rendements, et sont vecteurs de perfectionnement au stade agricole.

Fille de la culture (sucrerie) ou de l'élevage (laiterie), l'industrie agricole contribue à la transformation de la vie à la ferme (Bobin, 1926) et s'affirme comme source de richesse indéniable et incontournable à l'échelle de régions agricoles de plus en plus vastes.

\section{Quel système de relation entre les agriculteurs et les agro- industries?}

Si l'on excepte les travaux sur les fruitières surtout, le mode relationnel entre agriculteurs et industriels est peu présent dans les écrits analysés. Il figure surtout au titre du principe de la contractualisation, qui consiste en la fixation des prix ou du volume à livrer. Pratiquée de longue date dans l'industrie des conserves de légumes comme soupape de sécurité, régulateur dans un marché des denrées périssables, les conditions paraissent plutôt favorables pour le producteur, ce qui s'explique par un manque relatif de matière première (petits pois, haricots) (Sorre, 1950b).

Le cadre est tout autre entre la sucrerie et le planteur de betteraves puisque ce dernier est soumis au respect d'un contingentement (Sorre, 1929). Le contrat est conçu conjointement par les représentants des planteurs (Commission interdépartementale betteravière) et des fabricants (Groupe de la région du Nord des Sucreries et Distilleries) à l'échelle régionale (Nord-Pas-de-Calais). La précision des détails, du prix d'achat aux livraisons, reflète une certaine intégration verticale, l'exploitation devenant l'annexe de la sucrerie. Dans une note infra-paginale, R. Dion (1933) utilise aussi ce terme de quasi ou de semi-intégration, notant qu'» on la pratiquait avant d'avoir inventé le mot pour la culture du cornichon» alors qu'elle se diffuse pour l'aviculture et l'élevage de porcs auprès d'agriculteurs du Toulousain à la recherche d'une certaine sécurité.

Durant cette période de la fin du XIxe siècle aux années 1950 qui correspond à la phase d'émergence et de première structuration des agro-industries, ces dernières sont moins associées pour leurs activités propres que comme élément constitutif de la vie rurale locale, ce qui fait qu'elles arrivent de manière systématique en fin d'analyse. Malgré quelques travaux précurseurs, c'est surtout à partir des années 1960 que le regard porté par les géographes à leur égard évolue : d'objet d'étude géographique, elles deviennent objet géographique, et R. Brunet (1965) considère même que leur absence ou leur faiblesse est un handicap certain pour des campagnes toulousaines confrontées à un environnement en mutation.

\section{L'agro-alimentaire comme objet géographique}

21 Avec les années 1960 s'ouvre un «âge d'or " pour les IAA: l'urbanisation accélérée, l'évolution de la consommation alimentaire, la disponibilité de capitaux et l'aide publique permettent leur industrialisation et leur croissance. Pour autant, à la suite de J.-P. Peyon et F. Plet (1974) qui s'étonnaient de la faiblesse de la référence aux organismes d'aval à l'agriculture dans les publications géographiques depuis 1950, on ne peut que confirmer jusqu'aux années 2000. Pour la quarantaine de publications répertoriées, on doit souligner le rôle central de la revue Norois (9 articles entre 1957 et 1995) ${ }^{5}$. 


\section{Définitions et représentations des industries transformant des matières premières agricoles} industrie de transformation des produits agricoles pour G. Richez (1967), IAA pour R. Livet (1970), industries agricoles et alimentaires pour C. Cabanne (1973), l'agroalimentaire pour A. Fiette (1983), industrie agro-alimentaire pour F. Plet (1989), firmes agro-industrielles pour J.-P. Charvet et F. Plet (1996), etc. Cette diversité terminologique pour qualifier ces activités au fil du temps reflète parfaitement leur profonde hétérogénéité. En effet, de manière générale, les IAA associent des activités cloisonnées articulées verticalement tout en étant étrangères les unes aux autres (sucre, chocolaterie, etc.). Pour autant, parallèlement à la généralisation de l'expression «industries agroalimentaires » à partir des années 1970, un consensus prévaut pour une définition que l'on doit aux travaux d'un économiste, L. Malassis (1979), qui y regroupe l'» ensemble des entreprises transformant des produits, en général d'origine agricole, pour satisfaire les besoins alimentaires des consommateurs ».

La difficulté à cerner avec précision les activités couvertes explique pour partie le manque d'intérêt qu'elles suscitent chez les spécialistes de géographie industrielle. Certains pointent même un besoin impérieux de clarification au moins à des fins statistiques d'autant que les services ministériaux (ministère de l'Agriculture - et non ministère de l'Industrie -, services de la comptabilité nationale) ne s'appuient pas sur une liste identique, ce que pointait déjà C. Cabanne en 1973.

sources statistiques les présentent: l'Enquête Annuelle d'Entreprises (EAE) du ministère de l'Agriculture, et l'UNEDIC. Prenons la plus utilisée, l'EAE. Inclues dans la nomenclature d'activités française (NAF) depuis 1993, les IAA y sont subdivisées en 9 ensembles (NAF 15.1 à 15.9) et 41 sous-ensembles, avec des regroupements " par affinités ${ }^{6}$ » qui ont pour inconvénient leur variation dans le temps ${ }^{7}$, ce qui interdit la comparaison par séries longues. Outre les catégories d'activités, les seuils aussi interpellent : ne sont comptabilisées depuis 1998 que les entreprises de plus de 20 salariés (contre 10 auparavant, sauf pour la coopération) ou ayant plus de 35 millions de francs de chiffre d'affaires, ce qui minimise leur nombre, l'emploi et la réalité de leur participation à l'économie générale (Chalmin, 1983) dans le cadre d'une étude régionale (Plet, 1989; Poulot et Rouyrès, 2004).

À partir des années 1960, la mise à disposition de données couplée d'un souci de description statistique des faits géographiques, induit un recours plus fréquent à une cartographie intégrée dans les thèses ou articles, et dans les atlas qui tendent alors à se multiplier. En général, les cartes thématiques produites reposent sur un traitement sommaire des données. La simple représentation de la localisation sur les cartes de différents atlas régionaux démontre ainsi d'une certaine manière la faible place que tiennent ces activités pour les concepteurs. Souvent, le figuré ponctuel les représentant se surajoute aux à-plats des activités agricoles, quant à elles nettement plus détaillées grâce à l'exploitation du Recensement Général de l'Agriculture à partir de 1955.

26

Dans leur bilan d'une décennie de géographie rurale « quantitative et théorique ", V. Rey et M.-C. Robic (1983) notent que «les chercheurs qui travaillent sur les domaines mal saisis par l'information statistique ou ayant des données "qualitatives" sont handicapés pour utiliser l'arsenal actuel ». Et de citer l'agro-alimentaire pour laquelle, si on relève 
une certaine schématisation et une quantification, la tendance n'est pas à la réalisation de typologies, encore moins de "modèles ». En fait, les cartes lissées réalisées par F. Plet (1993) ou les cartes de C. Canevet (1993) matérialisant les dynamiques spatiales des entreprises agro-alimentaires bretonnes se démarquent de l'ensemble par l'objectif revendiqué de démonstration d'un processus spatio-temporel du changement.

\section{Une géographie des IAA en perpétuel mouvement}

À l'échelle de la France, de 1950 à 1980, le dynamisme agro-alimentaire est paysan (Chalmin, 1983) :conduit parallèlement au processus de spécialisation agricole, il a reposé sur une migration des sites industriels des centres de consommation vers les zones de production spécialisée (industrie de la viande). La dynamique s'inverse ensuite. La proximité de grands marchés de consommation oriente les choix de localisation, en particulier pour les moyens et grands établissements dans les secteurs de la consommation de masse. La réurbanisation partielle des activités (Plet, 1993) consacre une banalisation des stratégies industrielles, qui ne se différencient plus de celles des autres secteurs.

En conséquence se dessine une géographie agro-alimentaire où compte prioritairement un facteur " proximité-distance » basé prioritairement sur des critères d'accessibilité aux lieux de consommation ou de marché de main-d'œuvre (Vergneau, 1988). Ce constat varie cependant selon les régions: réel pour l'île-de-France (Plet, 1989) ou le Nord-Pas-deCalais (Vaudois, Le Mené, 1989) où la localisation est avant tout un héritage de la fin du xIxe siècle, il l'est moins en Picardie, à l'exception des villes les plus proches de Paris (Beauvais, Compiègne) (Fiette, 1985). En corollaire, l'absence d'un maillage urbain suffisant peut s'avérer disqualifiant. Première région céréalière pour sa récolte de blé, la région Centre n'est pourtant classée qu'au $5 \mathrm{e}$ rang national pour les effectifs salariés de ses établissements spécialisés dans le travail du grain.

Cette géographie en perpétuel mouvement surtout à partir des années 1980 résulte de phases de fermetures de sites partiellement compensées par de moins nombreuses créations en site urbain ou périurbain, ces dynamiques ayant accentué le phénomène de déséquilibre entre régions "favorisées » et " défavorisées », les régions les mieux dotées en équipements productifs n'étant pas épargnées. En Bretagne, la rupture dans la croissance des effectifs salariés date de 1982 (Canevet, 1993), et en région parisienne, le contexte est non seulement au déclin global de l'activité productive, mais aussi du rôle de commandement, la région devenant une vitrine de l'agro-alimentaire national et international (Plet, 1989 ; Poulot et Rouyrès, 2004).

La simplification du tissu productif induit des modifications dans le mode de structuration de l'espace par l'activité industrielle. En particulier se pose la question de la localisation du centre décisionnel car de nombreuses restructurations ont entraîné l'arrivée de capitaux étrangers (rôle de premier plan pour Nestlé), avec un risque de fragilité ultérieure en cas de retrait. À propos du groupe Perrier, C. Canevet (1993) parle d'» intrusion relativement brutale et spectaculaire du capital extérieur, perçue à l'époque comme une colonisation économique ». Ayant aussi recours au terme de colonisation pour l'industrie de la conserve en Picardie, A. Fiette (1985) note que, « incomplètement solidaire de la trame agricole, l'activité agro-alimentaire n'est thiérachienne qu'en figuration", ce qu'il explique par la nature des produits transformés et le poids des capitaux extérieurs. Or, ce dernier point est souvent crucial dans la mesure où le capital 
extérieur est plus mobile, plus opportuniste et plus exigeant, même dans les régions où ont été opérées de fortes économies d'échelle comme en Bretagne (Canevet, 1993).

\section{Des laiteries aux industries laitières : des noms emblématiques}

31 "À propos de l'évolution de la géographie industrielle en France », A. Fischer (1996) remarque qu'au tournant des années 1960 se dégage une étape structurelle dominée par les études sur les entreprises et groupes, par la prise en compte de leurs structures sociales et financières, par l'attention apportée aux analyses des stratégies géographiques. De nombreux géographes étant marxistes, leur attention va à la composition du capital des firmes, au jeu des prises de participation et aux mécanismes qui assurent à certains groupes le contrôle de pans entiers d'industrie. Déjà perceptible durant l'entre-deux-guerres, cette orientation est le fait à partir des années 1960 de géographes explorant le monde laitier. Les méthodes sont souvent empiriques, nécessitant un véritable travail de fond pour accéder aux sources des entreprises notamment.

Les travaux de M.-R. Daudé (1966) et d'A. Frémont (1967) sont représentatifs d'une focalisation sur des industries laitières en devenir dès 1929 avec G. Azambre. Ces trois auteurs s'appuient sur des critères techniques pour les différencier ${ }^{8}$, les techniques s'apparentant alors à un marqueur - spatial - de l'ouverture à un vaste marché pour la production rurale. Charnière du marché (Frémont, 1967), les industries profitent d'innovations techniques pour asseoir un marché de masse standardisé en place du marché local de haute tradition (beurre industriel en place du beurre de cru), pour partie sous l'influence de conceptions hygiéniques (Sorre, 1950b). Dès lors que la primauté va à la régularité et à l'adaptation aux besoins des consommateurs, on assiste à la rupture du lien au lieu entre le produit et le local (dès le début du xxe siècle pour le beurre) (Delfosse, 2002) et au passage d'un stade diffus à un stade de localisation pour les établissements. De fait, les industriels doivent faire face à de nouveaux comportements de consommateurs dont la conquête passe par la publicité notamment. Pour preuve, l'article de M. Chesnais (1972) qui, dès son introduction, rappelle que « la publicité associe les noms d'Elle-etVire, de Claudel, de Gloria ou de Gervais aux images de grasses prairies, de vaches opulentes, de crus laitiers de grande qualité ».

33 Cette image assignée à l'industrie influe sur les orientations de recherche. Même si leur généralisation génère des faillites ou des rachats d'autres laiteries, non sans répercussions sur l'économie agricole locale, la grande industrie est plutôt perçue comme un outil fort d'aménagement du territoire au moins jusqu'à la fin des années 1980. En effet, par effet induit, elle participe de la modernisation agricole (Brunet, 1965) pour partie en raison du rôle joué par un nouvel acteur, indépendant ou salarié de l'entreprise, le « ramasseur » (Daudé, 1966). Son rôle s'impose d'autant que les zones de ramassage du lait tendent à s'élargir, ce qui nécessite une gestion très fine (Henniquau, 1972).

Multipolaire, la grande industrie contribue à la diffusion d'un mode de fonctionnement basé sur la division du travail, ce qui aboutit à une nouvelle structuration sur site et entre sites. Ainsi, alors que les sites sont classiquement spécialisés sur un produit donné (beurrerie, fromagerie), fréquemment doublé d'un ou de deux secondaires (Daudé, 1966), on assiste courant des années 1950 à l'émergence de centrales polyvalentes. En fait, dans leur recherche d'une meilleure efficacité, les firmes optent entre deux orientations : soit elles associent des unités spécialisées à grande capacité de production, des flux de 
matières premières matérialisant ce lien; soit elles concentrent les fabrications dans une centrale polyvalente de grande taille approvisionnée en lait par des centres de collecte (Frémont, 1967). Une cartographie représente parfois cette géographie des flux (Lartaut, 1961).

Véritable révolution dans la vie rurale, l'industrialisation de la transformation du lait n'a été rendue possible que grâce à la mobilisation des hommes et d'importantes capacités financières. On voit alors se multiplier les organigrammes de présentation de groupes qui ne reflètent en général aucune attache géographique autre que, éventuellement, dans les dénominations des firmes.

Les groupes laitiers du secteur privé ont peu retenu l'attention des géographes, à l'exception notable du travail novateur d'A. Frémont sur la holding Gervais, dont la puissance financière lui autorise une politique de contrôle direct du circuit de commercialisation de ses produits.

Élément organisateur du monde rural, les coopératives ont donné lieu à une littérature abondante ${ }^{9}$, surtout quelques réussites notoires du grand Ouest de la France. «Fait géographique intéressant» (Leherpeur, 1957), la beurrerie coopérative d'Elle-et-Vire devient un "empire » pour A. Frémont (1967). Pour autant, à part la présentation de Dauphilait (Martin-Noël, 1962), il faut attendre la thèse d'État de J.-P. Peyon (1983), première synthèse $d u$ fait coopératif à l'échelle nationale, pour que des structures d'envergure soient analysées (Sodima-Yoplait, etc.), la décennie 1990 marquant un renouveau des recherches à ce sujet : l'UnionLaitière Normande (Sebille, 1989), Even (Calvez, 1997) ou Sodiaal (Le Mené, 1994). Toute une production cartographique y est associée.

Pourquoi cet intérêt particulier pour les beurreries coopératives? Industrie née au Danemark dès les années 1860 sous forme coopérative, en France, le «modèle danois » est notamment "prôné par les agrariens de la fin du xixe siècle et du début du xxe siècle " (Delfosse, 2002). A. Demangeon (1929) et M. Sorre (1950b) ont une forte admiration pour ce système spatial qui envisage les stratégies (production, commercialisation) à l'échelle régionale et nationale. Et c'est à la connaissance du «secret danois » que L. Leherpeur (1957) attribue l'origine des coopératives laitières de la Manche.

39 Sur le plan géographique, les grandes firmes coopératives combinent trois organisations spatiales qui ne se recoupent pas toujours : un espace de collecte, un espace de travail propre à l'établissement et un espace commercial (Peyon, 1983). Chacune fait l'objet de stratégies spécifiques, la première ayant donné lieu à de nouvelles réflexions en termes de territoire de firme (Margetic, 2003).

Représentatives d'une "démocratie économique dans la civilisation industrielle " (Frémont, 1967), plusieurs auteurs (Peyon, 1983; Sebille, 1989; Canevet, 1993) s'interrogent sur leur capacité à maintenir une spécificité coopérative dans un contexte de forte concentration des outils industriels et commerciaux surtout à partir des années 1980 puis 1990. Progressivement, l'interrogation s'est ainsi déplacée de leur spécificité structurelle vers leur dynamique économique.

41 Originales par la dimension coopérative et dominées par le poids croissant des grandes firmes, les IAA sont avant tout abordées par des analyses empiriques. Dans leur synthèse quant à «la géographie rurale "quantitative » et "théorique » », V. Rey et M.-C. Robic (1983) mettent d'ailleurs en avant la faible influence des travaux contemporains de F. Perroux sur les pôles de croissance, ou de W. Isard sur les activités motrices. Par contre, 
l'élargissement constant des espaces d'action des agro-industries oblige en quelque sorte les géographes ruralistes à s'adapter à de nouvelles échelles de la réalité.

\section{L'agro-alimentaire moteur du dynamisme des bassins de production?}

Au tournant des années 1970, le rôle des entreprises ne cessant de croître, les géographes ruralistes vont progressivement insister davantage, mais finalement de façon convergente, sur les effets de la «verticalisation » économique (Sautter, 1986). En effet, les producteurs voient leurs liens avec les villes les plus proches se distendrent dans le même temps où ils se trouvent de plus en plus intégrés au marché national ou européen par l'intermédiaire de filières spécialisées. C'est encore le secteur laitier, et plus particulièrement l'industrie de lait de consommation, qui est pris comme référence pour démontrer la perte du rôle d'animateur de la ville pour les campagnes voisines.

\section{La ville puis l'industrie laitière au cœur du bassin laitier}

Parmi les produits laitiers, jusqu'à la fin des années 1940, le lait de consommation a dominé dans les articles des géographes. Ceux-ci abordent les types de relations établis entre les villes et les campagnes environnantes, et plus particulièrement les interdépendances liées à l'approvisionnement de la ville en lait, soit une approche en termes de bassin, «espace de production plus ou moins continu, drainé par une ville, éventuellement à grande distance»(Brunet, 1993). Pour autant, le terme de bassin « laitier » ne figure jamais dans les intitulés d'articles.

Dès le début du xxe siècle, la demande urbaine même à longue distance a plus de poids que les aptitudes naturelles ou les traditions quant à l'organisation des spécialisations laitières dans des régions agricoles même éloignées du noyau urbain (Langeron, 1930). Pôle de cristallisation et de commercialisation, la ville s'efface pourtant au profit de son aire d'influence dans l'esprit des auteurs, et les laiteries sont un acteur parmi d'autres de ce système spatialisé.

Tous les bassins laitiers s'organisent selon une logique de cercles concentriques, avec deux (Dijon) (Richard, 1929) à quatre (Paris) (Dubuc, 1938) zones ou auréoles en fonction à la fois du poids de la ville centre et des possibilités en termes d'acheminement du lait (modes de transport). Si cette structuration rappelle la zonation de J. H. von Thünen (1826) (dans Margetic, 2003), cet économiste n'est jamais cité, ni dans les articles, ni dans les thèses consultées. Or, comparativement à sa définition d'aires spécialisées, la relative liberté de localisation de la production laitière fait de cette activité l'une des moins contraintes du modèle, la spécialisation portant alors sur les types de fabrication.

La ville participe donc activement à l'organisation des spécialisations laitières dans des espaces plus ou moins éloignés, et le lait de consommation s'impose simultanément comme choix stratégique pour les éleveurs, les industriels (dépôts, usines de conditionnement) et les transporteurs (wagon-citerne isotherme, gares laitières à Paris), imposant parfois des discontinuités dans l'aire d'approvisionnement d'autres spécialités (fromages du plateau de Langres) (Richard, 1929), ou l'affranchissement des traditions agricoles et des habitudes régionales de consommation (Arque, 1950). 
47 Sur les cartes, les limites du bassin sont matérialisées par la localisation des dépôts jusqu'aux années 1970, par celles des laiteries et de leur bassin d'approvisionnement en lait ensuite ${ }^{10}$. Cette progression dans le mode de représentation cartographique est révélatrice d'un réel changement de perspective chez les géographes, qui doit à une évolution de la réglementation.

48 À partir de la promulgation de la loi du 27 juillet 1940, le terme de bassin laitier acquiert un caractère réglementaire : d'une inorganisation topographique du ramassage, on passe à des zones délimitées et exclusives par laiterie fixées par décret (Baret, 1952). La réorganisation autoritaire du ramassage industriel et l'obligation de pasteurisation du lait (loi du 27 août 1940) font émerger les industries comme acteur central du système spatialisé, même si la ville conserve une influence certaine, et en 1976, J. Boichard évoque «le bassin laitier d'une entreprise ». Plus ou moins étendu, plus ou moins continu, ce bassin est analysé en termes de dynamique externe (élargissement spatial) et interne (évolution du nombre de producteurs, etc.).

49 La main mise économique des laiteries sur le bassin sert de fil conducteur au chapitre d'A. Frémont (1967) intitulé «Des producteurs aux transformateurs: la bataille des bassins laitiers ». En raison des multiples enchevêtrements des aires de ramassage des établissements, l'espace de collecte ne peut être homogène, mais composé de la juxtaposition de bassins plus ou moins polarisés, à la puissance d'attraction variable ${ }^{11}$. Clé de lecture des rapports de force entre producteurs et transformateurs, le prix du lait reflète des concurrences locales fruit de spécificités géographiques (ceintures laitières autour des grandes villes, bassins de transformation) mais aussi historiques (pôle herbager de renom).

50 Accordant une large place au marché, A. Frémont se situe à la charnière des travaux de géographie régionale et des analyses de filière qui prennent une place de plus en plus importante dans les travaux des géographes à partir des années 1970.

\section{La spatialisation des filières agro-alimentaires : les bassins de production}

51 Les premiers travaux qui formalisent le rôle central joué par les agro-industries sur la dynamique agricole sont le fait d'économistes américains de l'Université d'Harvard, J. H. Goldberg et R. A. Davis qui ont forgé le concept d' «agribusiness » en 1957 (Malassis, 1979). Repris à partir des années 1960 en France, plutôt par les économistes, la question est plutôt de savoir comment l'insertion croissante de l'agriculture dans les circuits marchands peut prendre forme sans entraîner une dépendance ou une intégration trop forte de l'activité agricole vis-à-vis de l'industrie. Elle a débouché sur les notions de « filière » (Diry, 1987), de « complexe » et de « système » agro-alimentaires.

L'abondance des travaux - de la part des économistes - sur les filières procède, historiquement, du mouvement de quasi-intégration de la production agricole par son amont et son aval comme le retrace J.-P. Diry (1985) dans sa thèse. Bien que le terme ne soit pas encore employé, c'est l'aviculture et l'élevage porcin qui servent de référence pour les premières observations, bien que ce mouvement soit déjà perceptible au niveau des sucreries ou des conserveries de légumes... mais de manière moins « lisible » car il ne génère pas de formes physiques comparables aux bâtiments d'élevage ${ }^{12}$. Sauf exception (Moreau-Poulot, 1988; Le Mené, 1994), la clé d'entrée est toujours l'agriculture, une 
agriculture en voie d'industrialisation par le biais de la diffusion d'une économie contractuelle, plus ou moins contraignante, avec le risque pour les géographes marxistes, d'une dépendance technologique et d'une dépendance économique liée.

53 Apparue tout d'abord comme cadre explicatif, puis descriptif de l'évolution de l'agriculture insérée dans l'économie marchande, la notion de filière renvoie à un champ d'investigation ou à un outil d'analyse de la réalité économique d'un produit ou d'un groupe de produits d'origine agricole donné (filière lait, filière sucre, etc.). Chaque filière est confrontée à des problèmes spécifiques et comporte sa propre assise territoriale, ce qui conditionne la logique des études quant à leur fonctionnement, et explique la diversité quant à l'approche privilégiée (Vaudois, 1987).

Dans ce contexte, la place que les auteurs accordent aux agro-industries est très variable, fonction de son importance stratégique pour la totalité de la filière, des régions (Diry, 1987), mais aussi d'une éventuelle intervention d'acteurs du "système d'encadrement " (Vaudois, 1987). Deux filières sont particulièrement représentatives de l'interventionnisme politique : les filières sucre et lait.

55 À propos du système agro-sucrier, M. Moreau-Poulot (1988) le définit en tant que filière obligée puisque les deux principaux partenaires - planteurs de betteraves et fabricant de sucre - sont liés par des relations contractuelles découlant de la politique de contingentement de la production sucrière et betteravière instaurée depuis 1934, reconduite ensuite dans des règlements sucriers européens. Seul habilité à octroyer un volume de production aux planteurs, le fabricant occupe une position stratégique incontournable. De plus en plus dominée par de très grands groupes sucriers qui concentrent leur activité sur les sucreries les plus compétitives, la géographie de la filière connaît constamment des mouvements conséquents de simplification du tissu productif, accompagnés par une rétraction plus secondaire des zones de production. En octobre 2002, la reprise du groupe Béghin-Say par les planteurs et l'interprofession relance un mécanisme qui pourrait bien s'amplifier après 2006 à l'échelle européenne avec la remise en cause du Règlement sucrier. Dans ce cadre, en fonction des options privilégiées, c'est le devenir de certains outils industriels, et par là même la structuration des zones de production dans des régions comme l'île-de-France qui pourrait se trouver hypothéquée.

Dans la filière lait, la mise en place des quotas laitiers en 1984 a suscité un renouvellement des écrits (Canevet, 1993; Ricard, 1993 ; Le Mené, 1994; Plet, 1994). Rupture de fond quant aux modalités de fonctionnement de la filière puisque, brutalement, on passe d'un contexte de "fleuve blanc en crue " à une situation de contingentement, elle génère une concurrence exacerbée pour l'accès à la matière première, et ce à différentes échelles géographiques. Attribué à l'éleveur, le quota est cependant géré au niveau des acheteurs de lait. Fort de cette appropriation, les laiteries engagent alors des stratégies de fidélisation en direction de leurs producteurs livreurs, voire de livreurs d'autres laiteries. Outre l'instauration de relations entre ces deux acteurs de la filière, la restriction à l'accessibilité de la matière première se traduit, pour les industriels confrontés à d'autres enjeux (marchés de plus en plus concurrentiels, moindre soutien financier de la part de la Communauté européenne, etc.) par la définition de nouvelles stratégies de croissance. En particulier, les nouveaux équilibres territoriaux s'appuient sur un tissu industriel réduit, surtout en milieu rural, le tissu coopératif accélérant même sa restructuration à partir du milieu des années 1980 .

Restructurations, fusions, etc. «L'instabilité de la géographie des IAA met précisément la transformation des produits agricoles au centre d'un débat politique sur les modalités du 
développement rural» (Vergneau, 1988) alors même que les pouvoirs publics avaient reporté le centre de gravité des filières sur le maillon industriel fin des années 1960 . L'objectif est d'aller à la conquête des marchés internationaux, tout en dynamisant un maillon agricole de plus en plus dépendant et un milieu rural aux prises avec d'éventuelles délocalisations, donc des risques de déstabilisation de l'emploi local (Vaudois et Le Mené, 1989). Si ce choix politique se justifie ultérieurement, les agroindustries étant plutôt épargnées par la crise économique de la fin des années 1970, le constat fin des années 1990 montre une inefficacité d'ensemble. Pour partie, elle résulte d'une inadéquation croissante entre l'aire de fonctionnement des agro-industries, dont les limites transcendent plusieurs limites administratives, et la limite des territoires de proximité les plus actifs (commune, intercommunalité, région). Dans le même temps, de manière indirecte, les agro-industries sont contraintes par les effets de la PAC sur la structuration des systèmes agricoles. Ainsi, la réforme de la PAC de 1992 a favorisé la conquête de nouvelles aires d'expansion par les conserveries dans les régions de grande culture (Margetic, 2003).

Reflet du poids croissant joué par l'économie de marché, les agro-industries, privées ou coopératives, préfèrent travailler avec des exploitations d'une certaine taille, si possibles proches l'une de l'autre. "Afin de réduire les frais de collecte et de livraison, elles favorisent la concentration économique des exploitations et la concentration géographique des productions » (Diry, 1987). En conséquence, « on a vu émerger la notion de bassin de production de pair avec celle de filière agro-alimentaire ainsi qu'avec celle de représentation de l'organisation spatiale "optimale" du secteur" (Pilleboue, 2000). Agro-industries et territoires ruraux s'articulent donc au niveau d'un bassin de production ${ }^{13}$ qui comporte en règle générale plusieurs établissements de transformation des produits agricoles, les agro-industries les plus puissantes étant de plus implantées dans plusieurs bassins parfois éloignés les uns des autres (Diry, 1997), pour profiter d'avantages climatiques pour les conserveries par exemple.

59 La structuration spatiale d'un bassin donné est directement tributaire des modalités de spécialisation de chacun des acteurs, et de leurs formes de sociabilité dans le temps. De la sorte, la réceptivité des agriculteurs aux attentes des industriels est un critère important pour la compréhension de l'efficience d'un bassin : à la méfiance des Auvergnats répond l'entreprenariat des Bretons en production porcine (Diry, 1992), avec la réussite connue de ces derniers. Un même engagement a assuré l'assise d'une filière de pommes de terre industrielle dans la région Nord-Pas-de-Calais (Margetic, 2003). En définitive, les bassins les mieux organisés ont une origine paysanne (Diry, 1997), même si les initiatives émanent des entreprises privées dans l'aviculture (Canevet, 1993), le monde paysan ayant de toutes manières perdu ensuite de son influence, surtout à partir des années 1980.

L'observation d'une mise en concordance d'un territoire et d'une "mentalité » rurale a été le fait de C. Canevet (1993) à l'échelle de la Bretagne, seul auteur à se référer à un bassin agro-alimentaire. Par cette notion, il sous-entend à la fois une adéquation et une combinaison entre aire de production et aire de transformation, une certaine densité d'échanges, un réseau de relations, une cohérence spatiale, en définitive une aire fonctionnelle, des sous-bassins locaux étant structurés par quelques entreprises motrices, sans que n'émerge de réelle position dominante. L'adjectif accolé au terme de bassin souligne plus particulièrement le réseau de relations - financières - entre coopératives mais entre entreprises privées. Bâtis sur des éléments de filières, ce système agro- 
alimentaire est en mouvement et à géométrie variable, mais à fondement breton incontestablement.

61 Chez les géographes, l'étude des rapports entre agrofilières et espace géographique a donc été conduite selon deux angles (Vaudois, 1996). Soit une dimension géographique a été intégrée à l'étude économique des filières de production, cette dernière étant envisagée comme un système spatialisé (Diry, etc.). Soit ont été ciblées les stratégies des acteurs des agrofilières et leurs incidences sur les espaces géographiques produits, ceuxci renvoyant à un système territorial (Canevet, 1993). Cette dernière décennie, la deuxième approche a été privilégiée par ajout d'une nouvelle variable, la qualité.

\section{Des bassins de production de la qualité agro-alimentaire}

Perceptible depuis le milieu des années 1980, la transition agricole marque « le passage progressif de l'agriculture de la période industrielle aux formes plurielles d'une agriculture multifonctionnelle mise en place pour fournir des réponses appropriées aux aspirations de la société » (Delfosse et Vaudois, 2000). Tant pour les agriculteurs que pour les industriels, l'enjeu n'est plus de produire en quantité, ce qui remet en cause les modèles intensifs, dont le plus intensif de l'Europe Atlantique, le modèle breton! (Baudelle et Canevet, 2000). Désormais, il s'agit plutôt d'offrir des aliments de qualité répondant à une variété d'attentes de la part des consommateurs (Pilleboue, 1999). La prescription d'exigences qualitatives renouvelées par ces firmes étant source de réagencements spatiaux et territoriaux, en particulier au niveau $\mathrm{du}$ bassin d'approvisionnement en produits agricoles, le thème de la qualité est devenu pratiquement incontournable pour appréhender les nouvelles règles des rapports entre agro-industries et agriculteurs, entre agro-industries et territoires. Ainsi, par cette entrée de la qualité, c'est la question des processus de déqualification et de requalification des espaces productifs qui est posée.

Notion polysémique et évolutive, la " qualité ${ }^{14}$ " agro-alimentaire est une préoccupation ancienne, qui a pris très tôt une dimension régionale puis nationale avec la reconnaissance de signes officiels de qualité, dont l'appellation d'origine contrôlée qui a constamment suscité l'intérêt d'un grand nombre de géographes, sans doute parce qu'elle fait explicitement référence à la notion d'ancrage territorial. En effet, la typicité du produit repose à la fois sur les caractères d'un terroir (ou d'une combinaison de terroirs) et sur des savoir-faire hérités et transmis (Rieutort, 2004). Sur le plan géographique, la mise en place d'AOC suppose donc un lourd travail de délimitation et la reconnaissance d'un « espace » de production aux limites franches et d'échelle variable, et un consensus entre divers acteurs sociaux et politiques (Ricard, 1993). Depuis le début des années 1990, malgré le choix politique de durcissement des critères de définition des $\mathrm{AOC}$, on assiste à une profusion de dossiers de demande, pour une multiplicité de produits (charcuteries, carottes de Créances, foin de Crau, etc.), dont la liste s'allonge d'année en année..., plutôt du ressort d'acteurs de la France du Sud (Rieutort, 2004).

Emblématiques des AOC françaises, les premiers travaux sur les AOC fromagères datent de l'entre-deux-guerres (Delfosse, 1992) et visent à démontrer l'association entre chaque fromage et un genre de vie particulier. Ainsi, les régions montagneuses sont le cadre d'un mode particulier d'organisation de la transformation: les fruitières (Ricard, 1993). Historiquement, on les aborde à partir du concept de région économique constituée autour d'une spécialité agricole, concept qui sert le discours régionaliste (Delfosse, 1992) : 
pour G. Azambre (1929), le fromage de maroilles permet de tracer la limite entre la Thiérache et les Ardennes.

Interroger les $\mathrm{AOC}$ fromagères revient donc à s'interroger sur la notion du lien d'un produit à un lieu. Plus ou moins évident en fonction du compromis entre acteurs de la production, de la transformation et les institutionnelsn (Ricard, 1993), ce lien s'est progressivement distendu sous l'effet des pressions exercées par les grands groupes laitiers qui gèrent un portefeuille d'AOC (Bongrain, Lactalis) (Rieutort, 2004). Ceux-ci développant une stratégie de segmentation du produit, on aboutit à une organisation en sous-espaces emboîtés et hiérarchisés, et la notion d'aire perd de sa pertinence (Pilleboue, 1999).

Depuis plus d'une dizaine d'années, les enjeux des filières $A O C$ ne relèvent plus uniquement $\mathrm{du}$ volet économique, mais débordent sur des problématiques de patrimonialisation et de développement local (Delfosse, 2002) et C. Durbiano (2000) évoque un "système de l'olivier» en Provence. Pour autant, la multiplicité des signes officiels de qualité ou l'appropriation de certains « noms » par les acteurs locaux (Aubrac, Brie de Meaux, etc.) nuit à l'image d'une géographie de la qualité tant s'imbriquent les démarches, les échelles, etc. Si l'on ajoute les marqueurs émanant de la profession agricole (guide des bonnes pratiques d'élevage...) ou des industriels (Route du Lait ${ }^{\oplus}$ de Sodiaal, etc.) en voie de généralisation, qui reposent sur la notion de traçabilité (Margetic, 2004), on brouille un peu plus encore la lisibilité de ces qualités territorialisées pour les consommateurs.

En définitive, de manière accélérée cette dernière décennie, la conception de la qualité est passée de la mise en exergue d'une minorité d'agriculteurs et de territoires particuliers (qualité "héritée ») à la diffusion la plus large possible à une majorité d'agriculteurs et de territoires (qualité "construite" autour de ressources inédites autour de politiques de relances). Au final, le fait de lier la qualité de produits agroalimentaires à un territoire n'est pas un acte qu'on peut séparer de son contexte.

\section{Conclusion}

Depuis le début du xxe siècle, les géographes ruralistes ont diversement appréhendé les industries agro-alimentaires au contact du monde agricole, en fonction de leur positionnement stratégique respectif en particulier. Ainsi, c'est avant tout des années 1960 aux années 1980 que ces firmes sont individualisées comme objet d'étude propre, au regard de leur dynamisme économique et de pratiques spatiales qui tendent à remodeler le maillon agricole. Par contre, durant la première moitié du xxe siècle, puis de nouveau à partir des années 1970, elles ne sont plus abordées pour elles-mêmes mais au travers de leurs interrelations avec le monde agricole d'abord, avec les acteurs des bassins de production ensuite. C'est la matière première agricole qui sert alors de fil conducteur, avec un relatif renouvellement des questionnements depuis les années 1990. Le thème de la "qualité » agro-alimentaire devient central, dans un contexte de transition agricole qui remet en cause les logiques productivistes du modèle agricole dominant et qui passe, pour les industriels, par la construction de nouvelles territorialités. Ce dernier point pourrait constituer une piste de recherche fructueuse pour cette nouvelle décennie. 


\section{BIBLIOGRAPHIE}

AleXANDRE (A.), 1928. - « L'économie agricole de la Picardie orientale depuis la guerre », Annales de Géographie, $\mathrm{n}^{\circ}$ 205, p. 61-73.

ARBos (Ph.), 1932. - « La culture des céréales en Limagne », Annales de Géographie, n² 231, p. 297-300.

ARQUe (P.), 1950. - « La laiterie dans la région de Fumel », Revue Géographique des Pyrénées et du Sud-Ouest, p. 315-317.

AzAmbre (G.), 1929. - «L'industrie laitière en Thiérache et dans le Hainaut français », Annales de Géographie, 216, p. 561-576.

BARET (M.-T.), 1952. - « L'élevage des bovins et le lait en Bas-Dauphiné », Revue de Géographie Alpine, p. 421-457.

BarRet (C.), Charvet (J.-P.), Dupuy (G.), Sivignon (M.), 2000. - Dictionnaire de géographie humaine, Paris, Liris, $190 \mathrm{p}$.

BAudelle (G.), CANeVet (C.), 2000. - « Les mutations du système agricole le plus intensif de l'Europe atlantique : vers une diversification des campagnes bretonnes ", dans SoUMAGNE (J.) (dir.), Les nouveaux espaces ruraux de l'Europe Atlantique, Poitiers, Maison des Sciences de l'Homme et de la Société, p. 122-135.

Berger (M.), GilletTe (Ch.), Robic (M.-C.), 1975. - « L'étude des espaces ruraux en France à travers trois quarts de siècle de recherche en géographie ", dans Réflexions sur l'espace rural français. Approches, définitions, aménagement, Université de Paris I/ENS Fontenay-aux-Roses, p. 3-51.

BobIN (R.), 1926. - « La Gâtine de Parthenay », Annales de Géographie, n 197, p. 404-412.

BOICHARD (J.), 1976. - L'élevage bovin, ses structures et ses produits en Franche-Comté. Annales littéraires de l'Université, Les Belles-Lettres, Besançon, 536 p.

Bonnamour (J.), 1997. - « La géographie rurale pendant le dernier quart de siècle », Ruralia, 1, p. 81-110.

BRUNET (R.), 1965. - Les campagnes toulousaines. Association des Publications de la Faculté des Lettres et Sciences Humaines, Toulouse, $727 \mathrm{p}$.

-, 1993. - Les mots de la géographie : dictionnaire critique, Montpellier, Documentation Française/ Reclus, $518 \mathrm{p}$.

CABANnE (C.), 1973. - « Les industries agricoles et alimentaires dans la France de l'Ouest », Norois, vol. XX, p. 328-335.

CALVEZ (E.), 1997. - Coopératives et industriels laitiers du Nord-Finistère. Évolutions agricoles, diversifications industrielles et emploi local, Université de Nantes, thèse de Doctorat, $460 \mathrm{p}$.

CANEVEt (C.), 1993. - Le modèle agricole breton, Rennes, PUB, 397 p.

Chalmin (P.), 1983. - « La dimension régionale des industries agro-alimentaires - Première partie », L'Information géographique, n 47, p. 183-193. 
CHARLES (J.), 1979. - « La collecte laitière en Béarn et Pays Basque : concentration du capital, encadrement des producteurs ", Revue Géographique des Pyrénées et du Sud-Ouest, tome 50, n², p. 227-250.

CHARVET (J.-P.), PLET (F.), 1996. - « Espaces ruraux et stratégies des firmes agro-industrielles », dans jollivet (M.), eizner (N.) (dir.), L'Europe et ses campagnes, Paris, Presses de Sciences Po, p. 323-342.

CHESNAIS (M.), 1972. - « L'industrie laitière en Basse-Normandie. Vers des dimensions suprarégionales ", L'Information géographique, n 4, p. 179-190.

Chevalier (M.), 1952. - « La vie agricole et industrielle des Prépyrénées ariégeoises », Annales de Géographie, n³27, p. 371-375.

Coudoux (J.), 1957. - L'économie agricole du Bas-Boulonnais, Lille, Diplôme d'Études Supérieures de Géographie, 2 tomes, 325 p. + CV planches.

DAUDE (M.-R.), 1966. - « Les laiteries et l'industrie laitière toulousaine », Revue Géographique des Pyrénées et du Sud-Ouest, p. 345-365.

Delfosse (C.), 1992. - La France fromagère, Université de Paris 1, nouvelle thèse, 343 p. + annexes -, 2002. - Géographie rurale, culture et patrimoine, Lille, Habilitation à Diriger des Recherches, 312 p.

Delfosse (C.), VAudois (J.), 2000. - « Éditorial. Les dimensions et les enjeux territoriaux de la transition agricole ", Hommes et Terres du Nord, n 4, p. 189-191.

Demangeon (A.), 1905. - La Picardie et les régions voisines : Artois, Cambrésis, Beauvaisis, Paris, A. Colin, $496 \mathrm{p}$.

-, 1929. - « Les aspects actuels de l'économie internationale (deuxième article) », Annales de Géographie, n²12, p. 97-112.

DiON (R.), 1933. - Le Val de Loire, étude de géographie régionale, 752 p.

DiRY (J.-P.), 1985. - L'industrialisation de l'élevage en France, Économie et géographie des filières avicoles et porcines, Paris, Ophrys, $680 \mathrm{p}$.

-, 1987. - « Géographie des filières agro-alimentaires ", Revue de Géographie de Lyon, n 4, p. 285-290.

-, 1992. - « Industries agro-alimentaires et développement régional. L'exemple de l'Auvergne et de la Bretagne », France et Grande-Bretagne rurales, Publications de l'Université de Caen, Centre de Recherches sur l'Évolution de la Vie Rurale, 15, p. 19-25.

-, 1997. - «Filières agro-alimentaires et bassins de production dans les pays développés », Bulletin de l'Association des Géographes Français, nº 4, p. 434-443.

Dubuc (R.), 1938. - « L'approvisionnement de Paris en lait », Annales de Géographie, n² 267, p. 257-266.

DURBIANO (C.), 2000. - « L'oliveraie provençale, production de qualité et requalification territoriale », Méditerranée, $\mathrm{n}^{\circ}$ 3-4, p. 17-27.

FIETTE (A.), 1983. - « L'Agro-alimentaire en Thiérache de l'Aisne », Hommes et Terres du Nord, nº 4, p. 54-56.

-, 1985. - « L’Agro-alimentaire en Picardie », Hommes et Terres du Nord, n 4, p. 288-300. 
FISCHER (A.), 1996. - « À propos de l'évolution de la géographie industrielle en France », dans W ACKERMANN (G.) (dir.), Nouveaux espaces et systèmes urbains, SEDES, Paris, p. 241-245.

FlatRes (P.), 1946. - « Le pays nord-bigouden », Annales de Géographie, n² 298, p. 139-141.

-, 1968. - « Les travaux français de géographie rurale de 1964 à 1968 ", Annales de Géographie, $\mathrm{n}^{\circ}$ 424, p. 734-743.

-, 1972. - « La géographie rurale en France », Recherches géographiques en France, Montréal, Comité national français de géographie, p. 189-194.

Fremont (A.), 1967. - L'élevage en Normandie, étude géographique, Caen, Association des Publications de la Faculté des Lettres et Sciences Humaines de l'Université, 2 vol., 626 et 318 p.

FRUIT (J.-P.), 1988. - Les sociétés rurales en France. L'apport des recherches géographiques (1980-1987).

Centre de recherches sur l'évolution de la Vie rurale, Caen, Commission de Géographie Rurale/ Congrès UGI de Sydney, 57 p.

Henniquau (T.), 1972. - « Un exemple de coopération agricole "La Prospérité Fermière" », Hommes et Terres du Nord, $n^{\circ}$ 1-2, p. 27-51.

Hinnewinkel (J.-C.), 2004. - Les terroirs viticoles, origines et devenirs, Bordeaux, Éd. Feret, 228 p.

JUILLARD (E.), 1964. - « Géographie rurale française. Tableaux récents (1957-1963) et tendances nouvelles ", Économie Rurale, p. 46-70.

LANGERON (G.), 1930. - « Le ravitaillement en lait de la ville de Dijon », Annales de Géographie, p. 526-529.

LARTAuX (J.), 1961. - « Les laiteries de Vendée », Norois, vol. VIII, p. 337-347.

LEHERPEUR (L.), 1957. - « Les coopératives laitières de la Manche. Un exemple : la société coopérative agricole beurrière des vallées d'Elle-et-Vire et son usine de Condé-sur-Vire ", Norois, vol. IV, p. 257-268.

LE MENÉ (C.), 1994. - «IAA et territoires : les industries laitières du Nord-Pas-de-Calais face aux quotas laitiers », Université de Lille, thèse de Doctorat, $470 \mathrm{p}$.

Livet (R.), 1970. - « Où en sont les IAA ? », Éudes vauclusiennes, nº 4, juillet-décembre, p. 1-4.

MALASSIS (L.), 1979. - Économie de la consommation et de la production agro-alimentaire, Paris, Cujas, $437 \mathrm{p}$.

MARGETIC (C.), 2001. - «Vers une nouvelle territorialisation des agrofilières : quelques exemples en Nord-Picardie ", dans BERGER (A.) (dir.), Dynamique rurale, environnement et stratégies spatiales, Montpellier, CNRS/Université Paul-Valéry, p. 551-560.

-, 2003. - Agro-industries et territoires : recherches en Nord-Pas-de-Calais, Arras, Habilitation à Diriger des Recherches, 287 p.

MARTIN-NOËL (J.), 1962. - « Une grande coopérative laitière alpine : Dauphilait », Revue de Géographie Alpine, p. 659-664.

MOREAU-POULOT (M.), 1988. - Planteurs et sucriers français : les mutations exemplaires d'une filière classique, Thèse de doctorat de Géographie, Paris, 972 p.

PARIS (D.), VAUDOIS (J.), 1987 - « L'espace rural dans la bibliographie récente », Hommes et Terres du Nord, $\mathrm{n}^{\circ} 2$, p. 129-132. 
PEYON (J.-P.), 1983. - La coopération agricole en France :étude géographique des grands organismes coopératifs, Université de Paris I, thèse d'État, 2 tomes, 936 p.

Peyon (J.-P.), Plet (F.), 1974. - « L'aval de l'agriculture dans les publications géographiques françaises ", dans Équipe de Géographie Rurale du Laboratoire Associé de Géographie Humaine, Paris, Rapport n², p. 125-156.

Pilleboue (J.), 1999. - « Les produits agro-alimentaires de qualité : remarques sur leurs liens au territoire ", Sud-Ouest Européen, n 6, p. 69-83.

-, 2000. - « Les nouveaux bassins de production de la qualité agro-alimentaire : clarification ou confusion territoriale? ", Hommes et Terres du Nord, n 4, p. 202-216.

PLET (F.), 1989. - « L'industrie agro-alimentaire en Île-de-France : de l'usine à la vitrine », dans L AKотA (A.-M.), MiLELLi (C.) (coord.), Île-de-France : un nouveau territoire, Paris, La Documentation française/GIP-Reclus, p. 127-148.

-, 1993. - « L'espace agro-alimentaire français : vers une rurbanisation partielle », dans REY (V.) (dir.), Géographies et Campagnes, Saint-Cloud, ENS Fontenay-aux-Roses, p. 99-108.

-, 1994. - Un itinéraire dans les champs de la géographie. Agriculture et agro-industries, espaces ruraux, régions, Université de Paris VIII, Habilitation à diriger des recherches, 8 vol., n.p.

Poulot (M.), Rouyres (Th.), 2004. - « L'île-de-France agro-alimentaire : vers de nouvelles relations territoriales entre IAA et agriculture ? ", dans MARGETIC (C.) (dir.), Dynamiques agro-industrielles et dynamiques rurales, Arras, Presses Universitaires d'Artois, coll. « Géographie », p. 109-131.

RAY (G.), 1948. - Les industries de l'alimentation, Paris, PUF, coll. « Que sais-je ?», 147 p.

Renaudin (C.), 1936. - « La vie rurale dans le nord de la Champagne », Annales de Géographie, ${ }^{\circ}$ 255, p. 235-239.

ReVerseau (G.), 1925. - « Les industries laitières dans les Charentes », Annales de Géographie, $\mathrm{n}^{\circ}$ 189 , p. 210-218.

Rey (V.), Roвic (M.-C.), 1983. - « La géographie rurale "quantitative" et "théorique" : bilan d'une décennie ", Annales de Géographie, n511, p. 305-330.

RICARD (D.), 1993. - Les montagnes fromagères en France. Terroirs, agriculture de qualité et appellations d'origine, Clermont-Ferrand, CERAMAC, $496 \mathrm{p}$.

-, 2002. - Les filières agro-alimentaires de qualité : recherches géographiques, Clermont-Ferrand, Habilitation à Diriger des Recherches, CERAMAC, $257 \mathrm{p}$.

RICHARD (M.), 1929. - « L'évolution agricole du Plateau de Langres », Annales de Géographie, $\mathrm{n}^{\circ} 212$, p. 133-147.

RICHEZ (G.), 1967. - « De quelques aspects de l'implantation d'une puissante industrie de transformation des produits agricoles dans le Gard », Méditerranée, p. 301-318.

RIEUTORT (L.), 2004. - « Filières d'Appellation d'Origine Contrôlée et territoires agro-industriels en France ", dans MARGETIC (C.) (dir.), Dynamiques agro-industrielles et dynamiques rurales, actes des Journées Rurales de la Commission de Géographie Rurale du Comité National de Géographie, Arras, Presses Université de l'Artois, p. 187-206.

SAUTteR (G.), 1986. - « La géographie rurale en crise ? ", Économie Rurale, 103-104, p. 259-274.

SEBILLE (A.), 1989. - Les IAA en Bretagne : dynamique des structures et développement régional, Université de Rennes, thèse de Doctorat, 1313 p. 
SORRE (M.), 1929. - L'agriculture et les industries agricoles, Lille, Éd. 1re Région Economique, coll. «Les ressources, l'outillage et la production de la Région du Nord », 109 p.

-, 1950a. - « La géographie des matières grasses », Annales de Géographie, n³14, p. 93-108.

-, 1950b. - Fondements de la géographie humaine, tome II : Les fondements techniques, vol II, Les techniques de production et de transformation des matières premières, A. Colin, Paris, $1031 \mathrm{p}$.

Sud-Ouest Européen, 1999. - « La qualité agro-alimentaire et ses territoires », Toulouse, Presses Universitaires du Mirail, $n^{\circ}$ 6, p. 1-102.

ThumeRelle (P.-J.), 1998. - « Un siècle de recherche en géographie humaine à Lille », Hommes et Terres du Nord, $\mathrm{n}^{\circ}$ 2, p. 77-83.

VAUdoIs (J.), 1987. - « Les filières agro-alimentaires face aux mutations de leur environnement : la dynamique récente des filières fleurs et légumes de serre aux Pays-Bas ", Revue de Géographie de Lyon, $\mathrm{n}^{\circ}$ 5, p. 387-410.

-, 1996. - «Économie de filière et dynamique des territoires : les bassins endiviers de la France du Nord », dans DonNAY (J.-P.), CHEVIGNE (C.), Recherches de géographie Humaine. Hommage au Professeur Charles Christians, Liège, Société Géographique de Liège, p. 237-243.

-, 2000. - « Les dynamiques spatiales des productions légumières : l'évolution récente des bassins endiviers de Nord-Picardie ", Méditerranée, n 3-4, p. 65-74.

VAudois (J.), Le MenÉ (Ch.), 1989. - « Évolution récente des industries agro-alimentaires dans la région Nord-Pas-de-Calais ", dans Paris (D.) (dir.), Le Nord-Pas-de-Calais, une région d'Europe en mouvement, Paris, La Documentation Française/GIP-Reclus, coll. « Dynamiques du Territoire », p. 91-142.

VERGNEAU (G.), 1988. - « Le remodelage de la géographie des IAA en France », Bulletin de l'Association des Géographes Français, n² 2, p. 139-150.

Veylet (P.), 1997. - « Les industries alimentaires au XIXe siècle », dans MARSEILle (J.) (dir.), Les industries agro-alimentaires en France : histoire et performances, Paris, Le Monde Édition, p. 39-40.

ZEYL (R.), 1930. - « La culture du houblon en Alsace », Annales de Géographie, n² 221, p. 569-578.

\section{NOTES}

1. Cet article fait suite à une communication lors du colloque « Faire campagne » (Rennes, 17-18 mars 2005).

2. Parmi les nombreux travaux sur le vin, on pourra se référer à J.-C. Hinnewinckel (2004).

3. E. Juillard (1964), P. Flatrès (1968, 1972), J.-P. Peyon et F. Plet (1974), M. Berger et al (1975), V. Rey et M.-C. Robic (1983), G. Sautter (1986), J.-P. Fruit (1988), J. Bonnamour (1997).

4. M. Berger et al., (1975) relèvent que « la plupart des analyses portant sur les activités autres qu'agricoles dans les campagnes françaises mettent l'accent sur leur liaison avec le système de production agricole" et de citer entre autres "les activités de transformation de produits agricoles (sucreries, distilleries, etc.) ».

5. À l'inverse de la revue Hommes et Terres du Nord créée en 1963 dont seuls cinq articles abordent directement les IAA. Ce thème n'est d'ailleurs pas repris par D. Paris et J. Vaudois (187), ce qui dénote l'affaiblissement progressif de cet axe de recherche au sein du laboratoire de géographie humaine après le départ de $M$. Sorre

6. Ces 9 ensembles sont les suivants: Industrie des viandes; Industrie du poisson; Transformation de fruits et légumes ; Industrie laitière ; Travail du grain, Fabrication de produits 
amylacés ; Fabrication d'aliments pour animaux ; Industries alimentaires diverses ; Industries des boissons (SCEES).

7. Par rapport à la classification d'activités et de produits (NAP) de 1973, l'industrie du poisson s'individualise alors que la préparation de plats cuisinés disparaît ; la fabrication industrielle de pain devient un sous-ensemble du travail du grain; la biscuiterie quitte la meunerie mais la malterie rejoint la brasserie. À l'inverse, sont créées de nouvelles subdivisions, comme la fabrication d'autres produits laitiers.

8. G. Azambre (1929) donne trois formes à l'industrie laitière thiérachienne: la laiterie domestique, la laiterie industrielle et la " grande usine de Boué ». En région toulousaine, M.-R. Daudé (1966) distingue cinq catégories : le laitier-artisan, le laitier-fromager, le centre collecteur dépendant d'une grande laiterie, la laiterie de moyenne importance et la grande entreprise laitière. En Normandie, A. Frémont (1967) en repère trois principales: les coopératives, des entreprises industrielles de type familial et des groupes intégrés.

9. Des articles les appréhendent en propre (Leherpeur, 1957 ; Martin-Noël, 1962 ; Henniquau, 1972) ou comme exemple d'industrie (Reverseau, 1925 ; Lartaut, 1961 ; Daudé, 1966). Les thèses leur consacrent des parties de plus en plus conséquentes (Frémont, 1967 ; Peyon, 1983 ; Le Mené, 1994 ; Calvez, 1997).

10. De manière générale, sa délimitation donne rarement lieu à de réelles investigations dans les écrits répertoriés. Se démarque J. Charles (1979) qui fait un parallèle avec la notion de bassin versant de manière imagée (" "gave” de lait et ses bassins d'alimentation ») ou J. Boichard (1976) l'associant aux communes d'une vallée.

11. Pour différencier les bassins laitiers, A. Frémont (1967) croise des éléments relatifs aux producteurs de lait (densité laitière, répartition, pouvoir de contestation) et aux transformateurs (nombre, dynamisme de la concurrence, type de produit et de circuit de commercialisation, etc.). 12. Le terme "intégration » est rarement employé par les géographes au sujet de la filière lait, sauf dans un article de J. Charles (1979) dans lequel il évoque une arme à double tranchant, pouvant déboucher sur la grève comme dans le monde ouvrier.

13. Deux définitions représentatives. Pour J. Vaudois (2000) : «Espace géographique spécialisé, structuré par les flux entretenus entre les exploitations agricoles productrices, entre ces dernières et les acteurs économiques et institutionnels de la filière considérée, entre l'ensemble ainsi formé et les acteurs du territoire support». Pour C. Barret et al., (2000) : "Portion de territoire spécialisée dans une production agricole particulière ou dans un tout petit nombre de productions agricoles (bassin laitier, céréalier...). La spécialisation repose sur l'existence d'un nombre significatif d'exploitations agricoles elles-mêmes étroitement spécialisées en relation avec la présence de filières performantes de commercialisation ou d'industries agro-alimentaires de première transformation. "

14. Si une habilitation (Ricard, 2002) et une revue (Sud-Ouest Européen, 1999) comportent le terme «qualité » dans leur intitulé, un nombre élevé d'articles (Baudelle et Canevet, 2000 ; Margetic, 2001 ; Rieutort, 2004, etc.) et de thèses (Delfosse, 1992 ; Ricard, 1993, etc.) intègre aussi cette entrée. 


\section{RÉSUMÉS}

Quelle appropriation du thème de l'agro-alimentaire par les géographes des campagnes en France depuis le début $\mathrm{du} \mathrm{xx}^{\mathrm{e}}$ siècle ? En ne retenant que les industries agro-alimentaires au contact du monde agricole, hors vin, et une sélection d'articles et de thèses considérés comme représentatifs, trois phases s'individualisent. Une seule (années 1960-1990) les érige en tant qu'objet géographique alors que les deux autres les relèguent, soit comme identificateur de l'économie agricole dans un contexte d'agrocentrisme des travaux (fin XIX ${ }^{\mathrm{e}}$-années 1950), soit comme acteur d'agrofilières et de bassins de production, dans un contexte de transition agricole cette dernière décennie (années 1970-2000).

How have geographers specialised in the French countryside appropriated the agribusiness subject since the early 20th century? Considering only agribusinesses in touch with the agricultural world - excluding wine-growing - and a selection of articles and theses considered as representative, three different phases appear. One only (1960s-1990s) considers them as geographical objects, whereas the other two relegate them either as rural economy identifiers in a context of agri-centered works (late $19^{\text {th }}$ Century-1950s) or as actors in agri-business channels or production areas in a context of agricultural transition in the last decades (1970s-2000s).

\section{INDEX}

Mots-clés : agro-alimentaire, épistémologie

Keywords : agri-food industries, epistemology

Index géographique : France

\section{AUTEUR}

\section{CHRISTINE MARGETIC}

CESTAN - UMR 6590 CNRS ESO, Université de Nantes, Chemin de la Censive du Tertre, BP 81307, 44313 Nantes cedex 3, France, cmargetic@nordnet.fr 